\title{
Associated Charged Higgs Production with Heavy Quarks: SUSY-QCD Corrections
}

\author{
Stefan Dittmaier \\ Albert-Ludwigs-Universität Freiburg, D-79104 Freiburg, Germany \\ E-mail: stefan.dittmaier@physik.uni-freiburg.de
}

Michael Krämer

Institut für Theoretische Physik und Kosmologie, RWTH Aachen University, D-52056 Aachen, Germany

E-mail: mkraemer@physik.rwth-aachen.de

\section{Michael Spira*}

Paul Scherrer Institut, CH-5232 Villigen PSI, Switzerland

E-mail: michael.spira@psi.ch

\section{Manuel Walser}

Paul Scherrer Institut, CH-5232 Villigen PSI

and Institute for Theoretical Physics, ETH Zürich, CH-8093 Zürich, Switzerland

\begin{abstract}
The dominant production process for heavy charged Higgs bosons at the LHC is the associated production with heavy quarks. We have calculated the next-to-leading-order supersymmetric QCD corrections to charged-Higgs production through the parton processes $q \bar{q}, g g \rightarrow t b H^{ \pm}$and present results for total cross sections and differential distributions. The QCD corrections reduce the renormalization and factorization scale dependence and thus stabilize the theoretical predictions. We present a comparison of the next-to-leading-order results for the inclusive cross section with a calculation based on bottom-gluon fusion $g b \rightarrow t H^{ \pm}$.
\end{abstract}

RADCOR 2009 - 9th International Symposium on Radiative Corrections (Applications of Quantum Field Theory to Phenomenology),

October 25 - 302009

Ascona, Switzerland

\footnotetext{
*Speaker.
} 

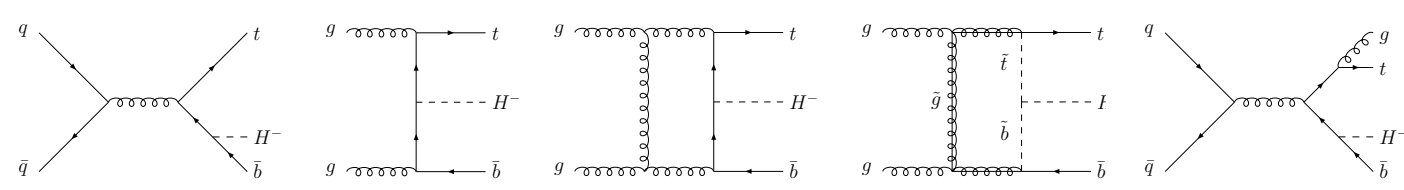

Figure 1: A generic set of diagrams for the Born level and examples of NLO diagrams.

\section{Introduction}

The Higgs mechanism [1] is a cornerstone of the Standard Model (SM) and its supersymmetric extensions. The masses of the fundamental particles, electroweak gauge bosons, leptons, and quarks, are generated by interactions with Higgs fields. The search for Higgs bosons is thus one of the most important tasks for high-energy physics and is being pursued at the upgraded proton-antiproton collider Tevatron with a center-of-mass (CM) energy of $1.96 \mathrm{TeV}$, followed by the proton-proton collider LHC with $14 \mathrm{TeV} \mathrm{CM}$ energy scheduled to start taking data in 2010.

The minimal supersymmetric extension of the Standard Model (MSSM) requires two Higgs doublets leading to five physical scalar Higgs bosons: two (mass-degenerate) charged particles $H^{ \pm}$, one CP-odd neutral particle $A$, and two CP-even neutral particles $h, H$. The discovery of a charged Higgs boson, in particular, would provide unambiguous evidence for an extended Higgs sector beyond the Standard Model. Searches at LEP have set a limit $M_{H^{ \pm}}>79.3 \mathrm{GeV}$ on the mass of a charged Higgs boson in a general two-Higgs-doublet model [2]. Within the MSSM, the charged-Higgs mass is constrained by the pseudoscalar Higgs mass and the W-boson mass through $M_{H^{ \pm}}^{2}=M_{A}^{2}+M_{W}^{2}$ at tree level, with only moderate higher-order corrections [3]. A mass limit on the MSSM charged Higgs boson can thus be derived from the limit on the pseudoscalar Higgs boson, $M_{A}>93.4 \mathrm{GeV}$ [4], resulting in $M_{H^{ \pm}} \gtrsim 120 \mathrm{GeV}$.

The LHC will extend the search for charged Higgs bosons to masses up to $M_{H^{ \pm}} \lesssim 600 \mathrm{GeV}$ [7], where the reach depends in detail on the values of the supersymmetric parameters. The most promising search channel for heavy $H^{ \pm}$(with $M_{H^{ \pm}} \gtrsim m_{t}$ ) at the LHC is the associated production of charged Higgs with heavy quarks, $p p \rightarrow t b H^{ \pm}+X$ (see Fig. 1).

Note that the inclusive cross-section prediction for the four-flavor-scheme (4FS) process $g g \rightarrow$ $t b H^{ \pm}$develops potentially large logarithmic terms $\propto \log \left(Q^{2} / m_{b}^{2}\right)$ which arise from the splitting of gluons into $b \bar{b}$ pairs, since the $b$-quark mass acts as a cutoff for the collinear singularity. The large scale $Q$ corresponds to the upper limit of the collinear region up to which factorization is valid. It has been argued that $Q$ is of the order of $M_{H^{ \pm}} / 4$ or less [8]. The $\log \left(Q^{2} / m_{b}^{2}\right)$ terms can be summed to all orders in perturbation theory by introducing bottom parton densities [9], provided that the $b$-quarks are produced predominantly at small transverse momentum. The LO process in this five-flavor-scheme (5FS) is charged Higgs-boson production through $g b \rightarrow t H^{ \pm}$. The first order corrections comprise the $\mathscr{O}\left(\alpha_{\mathrm{s}}\right)$ corrections to $g b \rightarrow t H^{ \pm}$and the LO process $g g \rightarrow t b H^{ \pm}[8]$.

\section{NLO Corrections}

In the following we review the 4FS calculation of the QCD and SUSY-QCD corrections to the parton processes $q \bar{q}, g g \rightarrow t \bar{b} H^{-}$in the MSSM [10]. The ultraviolet, infrared and collinear singularities have been regularized in $n=4-2 \varepsilon$ dimensions. The pentagon tensor integrals of 
the virtual corrections have been reduced directly to box integrals following Ref. [11] so that no inverse Gram determinants have been introduced which may spoil the numerical stability. Box and lower-point integrals have been reduced to scalar integrals using the standard Passarino-Veltman technique [12]. The real corrections have been calculated by applying the dipole subtraction formalism for the extraction of the infrared and collinear singularities [13]. Helicity amplitudes for the real matrix elements have been generated with MADGRAPH [14] and HELAS [15]. The phase space integration has been stabilized by implementing a multi-channel integration.

The strong coupling $\alpha_{s}(\mu)$ and the PDFs have been defined in the $\overline{M S}$ scheme with four active flavors, i.e. the SUSY particles and the top and bottom quarks have been decoupled from their running. The top and bottom quark masses have been defined on-shell, while the $\overline{M S}$ scheme has been adopted for the renormalization of the bottom-Higgs Yukawa coupling with decoupled sbottom-gluino contributions. The SUSY loops induce a modification of the tree-level relation between the bottom quark mass and its Yukawa coupling. The leading terms for large values of $\operatorname{tg} \beta$ can be summed to all orders [16] and absorbed in an effective Yukawa coupling. The corresponding terms have to be subtracted from the full NLO result in order to avoid double counting. The finite remainder of the genuine SUSY-QCD corrections turns out to be very small and thus negligible after this subtraction [10].

\section{Numerical Results}

The on-shell top mass has been set to $172.6 \mathrm{GeV}$ [17] and the bottom pole mass to $4.6 \mathrm{GeV}$ corresponding to a $\overline{M S}$ mass $\bar{m}_{b}\left(\bar{m}_{b}\right)=4.26 \mathrm{GeV}$. The Higgs mass calculation has been performed in the effective potential approach including corrections up to two loops [18] as implemented in the program HDECAY [19]. The strong coupling has been normalized to $\alpha_{s}\left(M_{Z}\right)=0.120$ in the calculation of the Higgs mass and the resummed $\operatorname{tg} \beta$-enhanced SUSY-QCD corrections to the effective bottom Yukawa coupling taking into account five active flavors. The scale of the strong coupling in these $\operatorname{tg} \beta$-enhanced terms has been chosen as the average of the sbottom and gluino masses in accordance with the recent NNLO results for these contributions [20]. The default renormalization/factorization scale of the running bottom mass, the strong coupling $\alpha_{s}$ and the PDFs has been chosen as the average of the final-state particle masses, $\mu=\left(m_{t}+m_{b}+M_{H^{ \pm}}\right) / 3$. The four-flavor strong coupling of the hadronic cross section has been evaluated with the QCD scale $\Lambda^{(4)}=0.347$ $\mathrm{GeV}$ at NLO and $\Lambda^{(4)}=0.220 \mathrm{GeV}$ at LO. We have used the MRST four-flavor PDFs [21]. For the numerical analysis we have adopted the SPS1b MSSM benchmark scenario [22] which is characterized by a large value of $\operatorname{tg} \beta=30$ and a correspondingly large production cross section of $p p \rightarrow t b H^{ \pm}$at the LHC.

The total LO and NLO cross sections for $p p \rightarrow t \bar{b} H^{-}$as well as the predictions involving a cut of $20 \mathrm{GeV}$ on the transverse momentum of the final-state bottom quark are displayed in Fig. 2 as functions of the charged Higgs mass. This process is dominated by the gluon-induced contribution at the parton level. The total $K$ factor is displayed in the lower part of the figure together with the error bands due to the scale dependence of the LO and NLO predictions. The $K$ factor is of moderate size over the whole range of charged Higgs masses. This moderate size, however, is induced by a significant cancellation of the pure QCD corrections of $\mathscr{O}(60 \%)$ and the genuine 

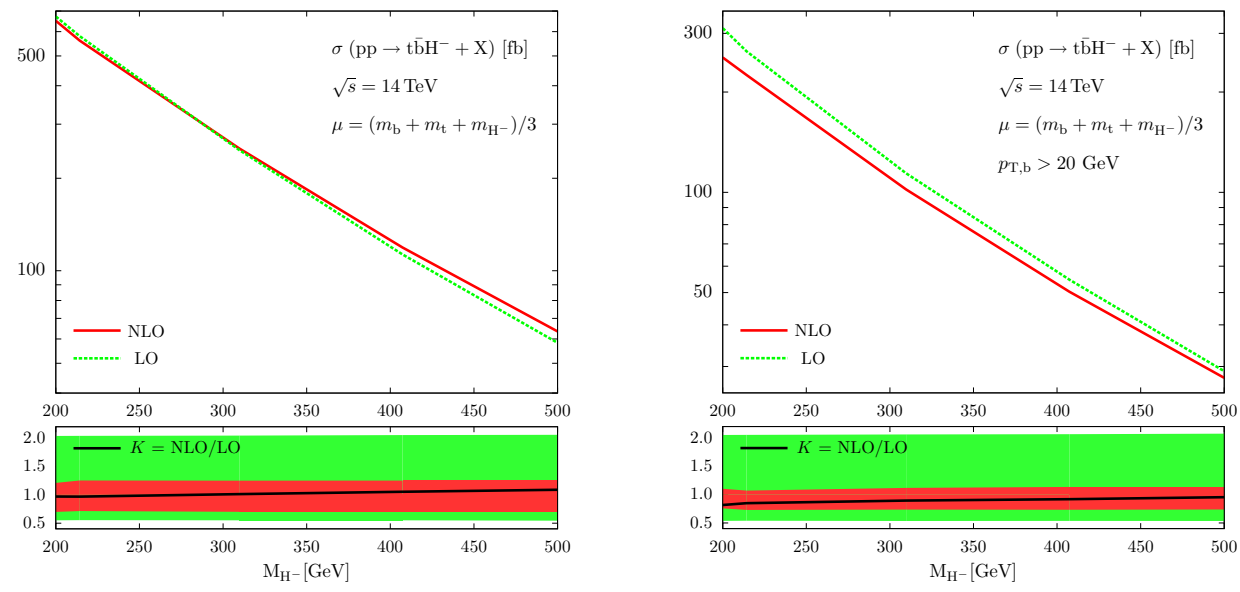

Figure 2: Total LO and NLO cross sections for $p p \rightarrow t \bar{b} H^{-}+X$ at the LHC as a function of the Higgs-boson mass, without (1.h.s.) and with (r.h.s.) a cut of $p_{T, b}>20 \mathrm{GeV}$ on the $b$-quark transverse momentum. The lower plots show the $K$-factor, $K=\sigma_{N L O} / \sigma_{L O}$, and the scale dependence of the LO and NLO cross section predictions for $\mu_{0} / 3<\mu<3 \mu_{0}$ normalized to the LO cross section with the central scale choice $\mu=\mu_{0}$.
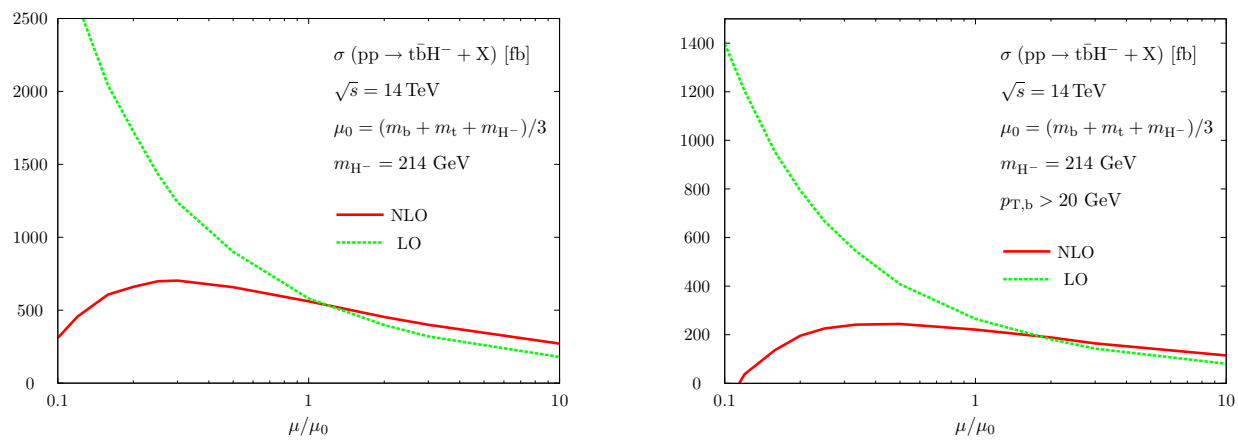

Figure 3: Variation of the LO and NLO cross sections with the renormalization and factorization scales for $p p \rightarrow t \bar{b} H^{-}+X$ at the LHC, without (1.h.s.) and with (r.h.s.) a cut of $p_{T, b}>20 \mathrm{GeV}$ on the $b$-quark transverse momentum.

SUSY-QCD corrections for the SPS $1 b$ scenario. Requiring the bottom quark to be produced with transverse momentum larger than $20 \mathrm{GeV}$ reduces the inclusive cross section by about $60 \%$.

The scale dependence without and with a transverse-momentum cut on the final-state bottom quark is shown in Fig. 3 as a function of the identified renormalization and factorization scales in units of the central scale $\mu_{0}=\left(m_{t}+m_{b}+M_{H^{-}}\right) / 3$ for a charged Higgs mass of $214 \mathrm{GeV}$. The scale dependences are significantly reduced from LO to NLO with a residual scale dependence of $\sim \pm 25 \%(\sim \pm 10 \%)$ without (with) a transverse-momentum cut.

In Fig. 4 we compare the transverse-momentum and rapidity distributions of the final-state particles, i.e. the top and bottom quarks as well as the charged Higgs boson. As expected the transverse-momentum distribution of the bottom quark is much softer than those for the top quark and the charged Higgs particle. However, there is a significant fraction of bottom quarks with transverse momentum larger than $25 \mathrm{GeV}$. The transverse-momentum distributions of the top quark and the charged Higgs boson are very similar with a maximum at $p_{T} \approx 100 \mathrm{GeV}$. The latter particles 

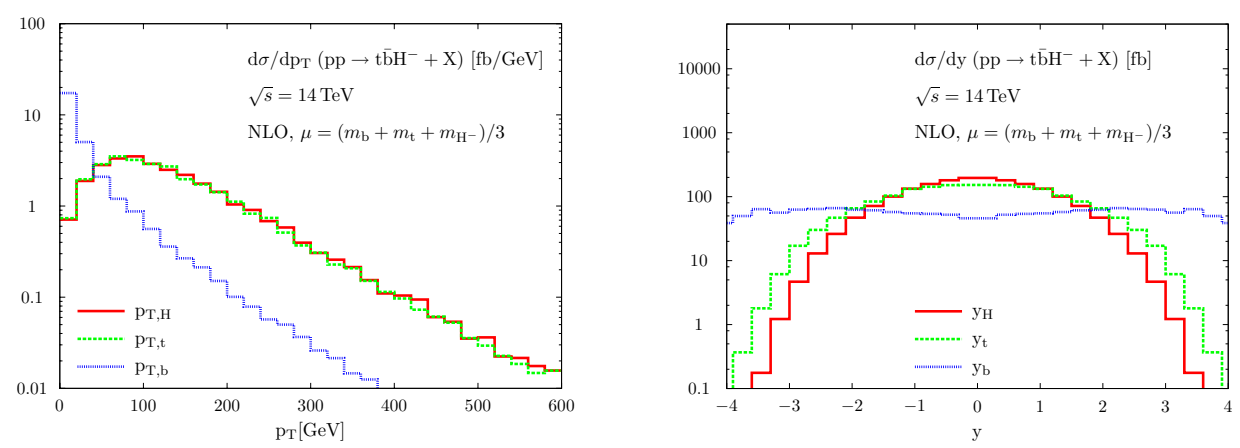

Figure 4: NLO transverse-momentum and rapidity distributions of the Higgs boson, the top quark, and the bottom quark for $p p \rightarrow t \bar{b} H^{-}+X$ at the LHC.

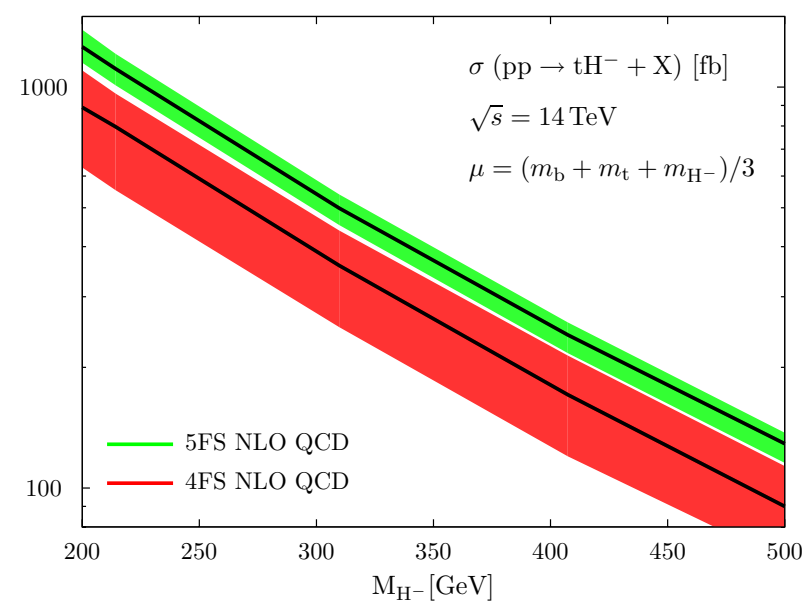

Figure 5: Total NLO cross section for $p p \rightarrow t H^{-}+X$ at the LHC as a function of the Higgs-boson mass in the $4 \mathrm{FS}$ and the 5FS. Shown is the central prediction and the scale dependence for $\mu_{0} / 3<\mu<3 \mu_{0}$.

are produced at central rapidities with $|y| \lesssim 2.5$, while the rapidity distribution of the bottom quark is rather flat in the region $|y| \lesssim 4$.

The impact of the NLO corrections on the shape of the transverse-momentum and rapidity distributions is different for the heavy particles, the top quark and charged Higgs boson, on one hand and the light bottom quark on the other hand. We find that the shapes of the top quark and Higgs transverse-momentum distributions are only mildly affected by the NLO corrections, while the shape becomes much softer from LO to NLO for the bottom quark. This effect originates from logarithmically enhanced contributions from collinear gluon radiation off bottom quarks. The NLO corrections do not change the shape of the rapidity distributions significantly at central rapidities $|y| \lesssim 2$.

Fig. 5 displays the comparison of our 4FS cross section with the corresponding 5FS cross section [8] including the pure QCD corrections at NLO, i.e. omitting the genuine SUSY-QCD corrections. The 5FS calculation has been evaluated with the five-flavor MRST2004 PDFs [23] and our set of input parameters and scale choices. The error bands indicate the theoretical uncertainties when varying the renormalization and factorization scales between $1 / 3$ and 3 times the central scale 
$\mu_{0}=\left(m_{t}+m_{b}+M_{H^{-}}\right) / 3$. Even taking the scale uncertainty into account, the 4FS and 5FS cross sections at NLO are barely consistent. The central predictions of the 5FS are larger than those of the 4FS by approximately 40\%, rather independent of the Higgs-boson mass.

\section{References}

[1] P. W. Higgs, Phys. Lett. 12, 132 (1964); Phys. Rev. Lett. 13, 508 (1964) and Phys. Rev. 145, 1156 (1966); F. Englert and R. Brout, Phys. Rev. Lett. 13, 321 (1964); G. S. Guralnik, C. R. Hagen and T. W. Kibble, Phys. Rev. Lett. 13, 585 (1964).

[2] A. Heister et al. [ALEPH Collaboration], Phys. Lett. B 543 (2002) 1.

[3] J. F. Gunion and A. Turski, Phys. Rev. D 39 (1989) 2701; A. Brignole, Phys. Lett. B 277 (1992) 313; M. A. Diaz and H. E. Haber, Phys. Rev. D 45 (1992) 4246; M. Frank, T. Hahn, S. Heinemeyer, W. Hollik, H. Rzehak and G. Weiglein, JHEP 0702 (2007) 047.

[4] S. Schael et al. [ALEPH Collaboration], Eur. Phys. J. C 47 (2006) 547.

[5] A. Abulencia et al. [CDF Collaboration], Phys. Rev. Lett. 96 (2006) 042003.

[6] V. M. Abazov et al. [D0 Collaboration], Phys. Rev. Lett. 88 (2002) 151803.

[7] ATLAS: Detector and physics performance technical design report, CERN-LHCC-99-014; G. L. Bayatian et al. [CMS Collaboration], J. Phys. G 34 (2007) 995.

[8] T. Plehn, Phys. Rev. D 67 (2003) 014018; E. L. Berger, T. Han, J. Jiang and T. Plehn, Phys. Rev. D 71 (2005) 115012.

[9] R. M. Barnett, H. E. Haber and D. E. Soper, Nucl. Phys. B 306 (1988) 697; D. A. Dicus and S. Willenbrock, Phys. Rev. D 39 (1989) 751.

[10] S. Dittmaier, M. Kramer, M. Spira and M. Walser, arXiv:0906.2648 [hep-ph]; Manuel Walser, disseration ETH Zürich NO. 17592, 2008

[11] A. Denner and S. Dittmaier, Nucl. Phys. B 658 (2003) 175.

[12] G. Passarino and M. Veltman, Nucl. Phys. B 160 (1979) 151.

[13] S. Catani and M. H. Seymour, Nucl. Phys. B 485 (1997) 291 [Erratum-ibid. B 510 (1998) 503];

S. Catani, S. Dittmaier, M. H. Seymour and Z. Trócsányi, Nucl. Phys. B 627 (2002) 189.

[14] J. Alwall et al., JHEP 0709 (2007) 028.

[15] H. Murayama, I. Watanabe and K. Hagiwara, KEK-91-11, January 1992 (unpublished).

[16] M. Carena, D. Garcia, U. Nierste and C. E. M. Wagner, Nucl. Phys. B 577 (2000) 88; J. Guasch, P. Häfliger and M. Spira, Phys. Rev. D 68 (2003) 115001.

[17] [CDF Collaboration], arXiv:0803.1683 [hep-ex].

[18] M. Carena, H.E. Haber, S. Heinemeyer, W. Hollik, C.E. Wagner and G. Weiglein, Nucl. Phys. B580 (2000) 29; H. E. Haber, R. Hempfling and A. H. Hoang, Z. Phys. C 75 (1997) 539.

[19] A. Djouadi, J. Kalinowski and M. Spira, Comput. Phys. Commun. 108 (1998) 56.

[20] D. Noth and M. Spira, Phys. Rev. Lett. 101 (2008) 181801 and arXiv:1001.1935 [hep-ph].

[21] A. D. Martin, W. J. Stirling and R. S. Thorne, Phys. Lett. B 636 (2006) 259.

[22] B. C. Allanach et al., Eur. Phys. J. C 25 (2002) 113 [eConf C010630 (2001) P125].

[23] A. D. Martin, R. G. Roberts, W. J. Stirling and R. S. Thorne, Phys. Lett. B 604 (2004) 61. 